Pure and Applied Mathematics Quarterly

Volume 3, Number 4

(Special Issue: In honor of

Grisha Margulis, Part 1 of 2)

$897-926,2007$

\title{
Affine Subdivision, Steerable Semigroups, and Sphere Coverings
}

\author{
Richard Evan Schwartz *
}

\section{Introduction}

Let $\Delta$ be a Euclidean $n$-simplex and let $\left\{\Delta_{j}\right\}$ denote a finite union of simplices which partition $\Delta$. We assume that the partition is invariant under the affine symmetry group of $\Delta$. A classical example of such a partition is the one obtained from barycentric subdivision, but there are plenty of other possibilities. (See §4.1, or else [Sp, p. 123], for a definition of barycentric subdivision.) Our partition gives rise to an affine subdivision rule for $\Delta$, which may be iterated. To subdivide each $\Delta_{j}$, we choose an affine map $A_{j}$ with $\Delta_{j}=A_{j}(\Delta)$, and then partition $\Delta_{j}$ into the collection $\left\{A_{j}\left(\Delta_{i}\right)\right\}$. The affine invariance of the partition translates into the fact that our partition of $\Delta_{j}$ is independent of the $(n+1)$ ! different choices for $A_{j}$. Now we iterate.

A basic question one can ask is Does the iteration of the subdivision rule produce a dense set of shapes of simplices? By shape of a simplex, we mean a simplex considered mod similarities. In $[\mathbf{B B C}]$ this question was raised and answered affirmatively for the case of 2-dimensional barycentric subdivision. In $[\mathbf{S}]$ we got the same result in 3 dimensions.

In general, a first step for the kind of density results just mentioned is as follows: Let $C_{n}$ be the collection of all $n$-dimensional simplices obtained by iteratively applying the subdivision rule. Let $\Omega_{n}$ be the collection of matrices of the

\footnotetext{
Received June 24, 2005.

* Supported by N.S.F. Grant DMS-0305047 and also by the Ruth Davis Foundation
} 
form

$$
\pm \frac{L}{|\operatorname{det}(L)|^{1 / n}}
$$

where $L$ is the linear part of an affine map from $\Delta$ to a member of $C_{n}$. Here, and always below, the sign is chosen to make the determinant positive. $\Omega_{n}$ is a semigroup of $S L_{n}(\boldsymbol{R})$, the group of $n \times n$ matrices of determinant 1 . To show that $C_{n}$ consists of a dense set of shapes it suffices to show that $\Omega_{n}$ is a dense semigroup in $S L_{n}(\boldsymbol{R})$.

The setup we have just described works for any subdivision rule of the type we have been considering, but henceforth we will concentrate on barycentric subdivision. Thus, $\Omega_{n}$ henceforth refers specifically to this case. Let $\widehat{\Omega}_{n}$ denote the group generated by $\Omega_{n}$. In $\S 5$ we will show easily that

Lemma $1.1 \widehat{\Omega}_{n}$ is dense in $S L_{n}(\boldsymbol{R})$ for any $n$.

So, we are left with the task of showing that $\Omega_{n}$ is dense in $\widehat{\Omega}_{n}$. Here is one easy result:

Lemma 1.2 If $\Omega_{n}$ has an infinite order elliptic then $\Omega_{n}$ is dense in $\widehat{\Omega}_{n}$.

Proof: Up to signs, $\Omega_{n}$ is generated by a single element $T_{1}$ and the group of affine permutations of the initial simplex. If $\Omega_{n}$ contains an infinite order elliptic element then this element must involve $T_{1}$. But then we can find an unbounded sequence $\left\{T_{1} w_{k}\right\}$ of words in $\Omega_{n}$ converging to the identity. Evidently $\left\{w_{k}\right\}$ converges to $T_{1}^{-1}$. Hence $T_{1}^{-1}$ lies in the closure of $\Omega_{n}$. But $\widehat{\Omega}_{n}$ is generated by $T_{1}$, $T_{1}^{-1}$ and the permutations.

Thus, to prove that $\Omega_{n}$ is dense in $S L_{n}(\boldsymbol{R})$ all we need is an infinite order elliptic element. In $[\mathbf{B B C}]$ and $[\mathbf{S}]$ some infinite order elliptic elements were found in $\Omega_{2}$ and $\Omega_{3}$ respectively and these were used as the basis for a density proof. However, the proofs there did not use Lemmas 1.1 or 1.2. For the interested reader, we will give 2 and 3 dimensional examples of infinite order elliptics in $\S 4.2$. Thus we get a different proof that $\Omega_{n}$ is dense in $S L_{n}(\boldsymbol{R})$ for $n=2,3$.

In the 3 dimensional case the infinite order elliptics seem quite rare, and we needed a computer search to find some. After a moderate amount of computer searching we haven't found any infinite order elliptics in $\Omega_{n}$ for $n \geq 4$; and we conjecture that there are none. Here we will develop a more robust method for showing that a semigroup $S$ is dense in the group $\widehat{S}$ it generates, and we will use the method to show that $\Omega_{4}$ is dense in $\widehat{\Omega}_{4}$. Hence 
Theorem $1.3 \Omega_{4}$ is dense in $S L_{4}(\boldsymbol{R})$ and so the iteration of 4-dimensional barycentric subdivision produces a dense set of shapes.

Our method could probably be adapted to work in a very broad setting of semigroups acting isometrically on unique geodesic metric spaces. However, we find that a lot of the apparatus for the method is already built into the case that our semigroup is a subset of a Lie group $G$ acting on a symmetric space $X$ of nonpositive curvature. In the case of affine subdivision, $G=S L_{n}(\boldsymbol{R})$ and $X=S L_{n}(\boldsymbol{R}) / S O(n)$. We will develop some of the theory for general pairs $(G, X)$ but will specialize to the choices above when appropriate.

We choose an origin $O \in X$. We say that $S$ is steerable if the orbit $S(O)$ contains a finite subset, which we call a steering wheel. A steering wheel has the property that any horoball containing $O$ in its boundary also contains a point of the steering wheel in its interior. See $\S 2.1$ for details. In $\S 2.2$ we will prove ${ }^{\dagger}$

Theorem 1.4 Let $S$ be a steerable semigroup of the isometry group of a nonpositively curved symmetric space. Then $S$ is dense in $\widehat{S}$.

To complete our proof of Theorem 1.3 we will prove

Lemma 1.5 $\Omega_{4}$ is steerable in $S L_{4}(\boldsymbol{R})$. Hence $\Omega_{4}$ is dense in $\widehat{\Omega}_{4}$.

We find our steering wheel using a computer search. After we find it, the problem of verifying the basic property in variable curvature is tricky. To help our cause, we will transplant the problem into hyperbolic space using the Hadamard map-i.e. geodesic polar coordinates. This is done in $\S 2.3$. We will prove a general comparison result which says that the set in $X$ is a steering wheel provided that the transplanted set in hyperbolic space is a steering wheel. In $\S 2.4$ we see that the problem we come to in hyperbolic space boils down to showing that a certain finite collection of balls covers the unit sphere in $\boldsymbol{R}^{\operatorname{dim}(X)}$. Hence the title of the paper. (In our example, $\operatorname{dim}(X)=9$.) Finally, we convert the sphere covering problem into an easily computable problem involving convex hulls of polytopes.

In $\S 3.1$ we give a formula for the metric in $X$. In $\S 3.2$ we provide the formula for the Hadamard map. The formula for the Hadamard map requires us to find the eigenvalues and eigenvectors of matrices. We would prefer to do calculations which are more straightforward than this, so in $\S 3.3$ we give some Taylor series

\footnotetext{
${ }^{\dagger}$ I'm pretty sure it follows from the simplicity of $S L_{n}(\boldsymbol{R})$ and the arguments in $\S 2.2$ that a steerable semigroup of $S L_{n}(\boldsymbol{R})$ is either dense or a cocompact lattice. I leave the details of this finer result to the interested reader.
} 
approximations to the Hadamard map. These approximations make our calculations transparant and allow us to work entirely with rational quantities or their square-roots. In $\S 3.4$ we include some Mathematica $[\mathbf{W}]$ code, which will let the interested reader play around with the Hadamard map and its approximations.

In $\S 4$ we will construct the steering wheel for $\Omega_{4}$. The steering wheel has 120 elements, each of which is the image of $O$ under an element of $\Omega_{4}$. Each of the 120 elements of $\Omega_{4}$ we use is the product of 36 generators! We found our steering wheel using a computer search, as we discuss in $\S 4.3$. One could think of the our search algorithm as a semigroup analogue of taking iterated commutators. Thus, in the end, our method is akin to the method used to prove the Margulis Lemma.

We prove Lemma 1.1 in $\S 5$ using explicit formulas for some commutator elements in $\widehat{\Omega}_{n}$. The formulas are rather beautiful and hint at some of the hidden algebraic structure of barycentric subdivision. The matrix calculations needed to justify the two main formulas are routine but tedious, and in the interest of space we will omit them. For smallish values of $n$, say $n \leq 10$, one can just perform the calculations explicitly. To this end, we have included a short Mathematica program which the reader can feed into a computer and operate easily. We really only care about the case $n=4$ in this paper.

In theory we could use our methods to prove Theorem 1.3 for larger values of $n$. However, the computational complexity of the problem rises quickly with the dimension. Maybe we could get up to $n=7$ without bringing in a supercomputer. An easier target for our methods would be other subdivision schemes in low dimensions. We might take this up in a future paper.

One major drawback of our method is that it only works one example at a time. However, if a semigroup is dense then it is also steerable and this can be detected by a finite amount of computation. Thus, any given example can be reduced to a problem about subgroups with our method, given a sufficiently powerful computer. As is usual with computer-aided mathematics, this state of affairs is not exactly ideal, but still is better than nothing.

I'd like to thank Sinai Robins for interesting conversations about polytopes, and also for his encouragement. I'd also like to thank Pat Hooper for discussions about convex hull algorithms. 


\section{Steerable Semigroups}

\subsection{Basic Definitions}

Let $X$ be a symmetric space of non-positive curvature. For convenience we assume that there is some pre-chosen "origin" of $X$, which we call $O$. In the cases of interest to us, there will be an obvious choice. Let $G=\operatorname{Is}(X)$, the isometry group of $X$. ${ }^{\ddagger}$ Let $S \subset G$ be a semigroup. We assume that $S$ is finitely generated, with generating set $s_{1}, \ldots s_{m}$. For any $w \in S$ let $|w|$ denote the minimum number of generators needed to express $w$ as a word.

We are interested mainly in the orbit $S(O)=\{s(O) \mid s \in S\} \subset X$. We make our multiplication correspond to the group action, so that

$$
s_{1} s_{2}(x)=s_{1}\left(s_{2}(x)\right) \quad \forall x \in X .
$$

A horoball in $X$ is the geometric limit of unboundedly large metric balls in $X$, provided that this limit is neither empty nor all of $X$. We say that a special horoball is one which contains $O$ in its boundary. Note that the set of special horoballs is compact. Here are our basic definitions.

Definition 1: Let $W \subset X$ be a finite subset. $W$ is a steering wheel if every special horoball contains at least one point of $W$ in its interior.

Definition 2: $S$ is steerable if $S(O)$ contains a steering wheel.

Lemma 2.1 Suppose $W$ is a steering wheel. Then there is some $R$ with the following property. If $B \subset X$ is a metric ball having radius at least $R$ and containing $O$ in its boundary then $B$ contains some point of $W$ which is at least $1 / R$ from $\partial B$.

Proof: This is really just compactness. If this lemma is false then we can find a sequence $\left\{B_{n}\right\}$ of counter-examples with the radius $R_{n}$ tending to infinity. Passing to a subsequence, we can let $B_{\infty}=\lim B_{n}$ be a horoball. (This limit cannot be all of $X$ because $0 \in \partial B_{\infty}$.) By construction $B_{\infty}$ is a special horoball which does not contain any point of $W$ in its interior.

\footnotetext{
${ }^{\ddagger}$ Technically, we are interested in subgroups of $S L_{n}(\boldsymbol{R})$, which is a slightly different group than $G$, when $X=S L_{n}(\boldsymbol{R}) / S O(n)$. However, the small discrepancy between the two groups never comes up and we will follow the common practice of just ignoring it.
} 
We define $R(W)$ as the smallest $R$ which satisfies the conclusions of Lemma 2.1. The following result says that essentially we can move near any point in $X$ following the orbit of a steerable semigroup. Let $d$ denote the distance function on $X$.

Lemma 2.2 Suppose $S$ is steerable, with steering wheel $W$. Let $R=R(W)$. Let $x \in X$ be any point and let $w_{1} \in S$ be any element. Then there is an element $w_{2} \in S$ such that $d\left(w_{1} w_{2}(O), x\right) \leq R$. Moreover, there is a universal constant $C$ such that $\left|w_{2}\right|<C d\left(x, w_{1}(O)\right)$.

Proof: Let $\sigma_{1}, \ldots, \sigma_{h} \subset S$ be a collection of elements such that $W=\bigcup \sigma_{j}(O)$. Let $y_{0}=w_{1}(O)$. If $y_{0}$ is within $R$ of $x$ we are done. Otherwise we proceed to construct points $\left\{y_{i}\right\}$ inductively. Suppose that $y_{i}=w_{1} v_{i}(O)$ has been constructed and $d_{i}=d\left(x, y_{i}\right)>R$. Here $v_{i}$ is a word in $\sigma_{1}, \ldots, \sigma_{h}$. Let $B_{i}$ denote the metric ball centered at $x$ and having radius $d_{i}$. Note that $y_{i} \in \partial B_{i}$.

We now note the following property of the set $w_{1} v_{i}(W)$, which follows from Lemma 2.1 and from the fact that $w_{1} v_{i}$ is an isometry of $X$ : Since $B_{i}$ has radius at least $R$ and contains $w_{1} v_{i}(O)$ in its boundary, there is some point $y_{i+1} \in w_{1} v_{i}(W)$ such that $y_{i+1}$ is contained in $B_{i}$ and at least $1 / R$ from $\partial B_{i}$. By definition, there is some index $j=j_{i}$ such that $y_{i+1}=w_{1} v_{i} \sigma_{j}(O)$. Hence $y_{i+1}=w_{1} v_{i+1}(O)$. Also, $d_{i+1} \leq d_{i}-1 / R$. Thus, for some $k \leq R d_{0}$ we must have $d_{k} \leq R$. We set $w_{2}=v_{k}$. Note that $\left|v_{i+1}\right| \leq\left|v_{i}\right|+\sup _{j}\left|\sigma_{j}\right|=\left\|v_{i}\right\|+C^{\prime}$. Hence $\left|w_{2}\right|<C^{\prime} R d_{0}<C d_{0}$.

\subsection{Proof of Theorem 1.4}

We will prove Theorem 1.4 through a series of smaller lemmas. To highlight an important fine point in the next lemma we introduce a bit of extra notation. Let $[S]$ denote the set of abstract words in the generators of $S$. Thus, an element of $[S]$ might be nontrivial but act trivially on $X$. (Think of the case when $S$ generates a discrete group.) Say that a word $w \in[S]$ is K-saturated if each generator of $S$ appears at least once in each length $K$ "block" of $w$. The empty word is $K$ saturated for every $K>0$. Say that $w$ is $R$-bounded if $d(O, w(O)) \leq R$. Here $R$ is the constant from Lemma 2.2. Let $\Omega(K)$ denote the set of words in $[S]$ which are both $R$-bounded and $K$-saturated.

Lemma 2.3 If $S$ is steerable then there is some $K$ such that $\Omega(K)$ contains infinitely many words. 
Proof: Let $w=s_{1} \ldots s_{m}$ be the product of the generators of $S$. Define $D=$ $d(w(O), O)$ and $K=C(D+R)+m$. Here $C$ is the constant from Lemma 2.2. Let $w_{0}$ be the empty word. Suppose that $w_{i} \in \Omega(K)$ has been constructed. We have $d\left(w_{i} w(O), O\right) \leq D+R$ by the triangle inequality. Applying Lemma 2.2 in the case when $x=O$, there is some word $w_{i}^{\prime}$ such that $\left|w_{i}^{\prime}\right| \leq C(D+R)$ and $w_{i+1}=w_{i} w w_{i}^{\prime}$ is $R$-bounded. By construction $\left|w_{i}^{\prime}\right| \leq K-|w|$ and $w$ contains each of the generators in order. Each $w_{k}$ in our infinite sequence consists of $k$ copies of $w$ separated by blocks having length at most $K-|w|$. From this it is easy to see that each $w_{i}$ is $K$-saturated. Hence $w_{k} \in \Omega(K)$ for all $k$.

Lemma $2.4 \Omega(K)$ contains an unbounded sequence $\left\{\beta_{k}\right\}$ of abstract words whose action on $X$ converges to the identity in $G$.

Proof: Note that the set of $R$-bounded elements is compact in $G$. Hence we can find a convergent sequence $\left\{w_{k}\right\} \in \Omega(K)$. Since $S$ is finitely generated, there are only finitely many choices for the first $n$ letters of $w_{k}$, independent of $k$. Hence, passing to a subsequence we can assume that there is a sequence of words $\left\{\beta_{j}\right\}$ such that $w_{k}=\beta_{1} \ldots \beta_{k}$ for all $k$. Passing to another subsequence, we can assume that $\left|\beta_{k}\right|$ is unbounded. By construction the words $w_{k}$ and $w_{k+1}$ have an action which differs by an arbitratily small amount. Hence $\left\{\beta_{k}\right\}$ converges to the identity word. Note that a subword of a $K$-saturated word is $K$-saturated. Hence $\beta_{k}$ is $K$-saturated.

Let $\left\{\beta_{k}\right\}$ be the sequence from the previous lemma. Since $\left|\beta_{k}\right|$ is unbounded but always $K$-saturated, and $S$ is finitely generated, there is some fixed word $w$, containing all the generators of $S$, which appears as the initial word of $\beta_{k}$ infinitely often. Let's label our generators so that $s_{1}, \ldots, s_{i}$ appear in $w$ before $s_{i+1}$ appears for each $i=1, \ldots, m$.

Let $w_{j} \subset w$ denote the longest initial subword of $w$ which does not contain $s_{j}$. Let $\gamma_{k}=w_{j}^{-1} \beta_{k} w_{j}$. By construction $\gamma_{k} \in S$ and $\gamma_{k}$ starts with $s_{j}$. We can write $\gamma_{k}=s_{j} \delta_{k}$, with $\delta_{k} \in S$, infinitely often. Since $\gamma_{k}$ converges to the identity $\delta_{k}$ converges to $s_{j}^{-1}$. Hence $s_{j}^{-1}$ lies in the closure of $S$. Now we know that the inverses of all the generators of $S$ lie in the closure of $S$. Now we know that $S$ is dense in $\widehat{S}$. 


\subsection{A Comparison Result for Steering Wheels}

Let $X$ be an $N$-dimensional symmetric space normalized so that its sectional curvature lies in $[-K, 0]$. Let $N=\operatorname{dim}(X)$. Suppose that $\boldsymbol{H}^{N}$ is hyperbolic space, normalized so that its curvature is $-K$. In this section we explain how to use $\boldsymbol{H}^{N}$ to verify that a given subset of $X$ is indeed a steering wheel.

Remark: In practice we will not compute the curvatures of $X$ and $\boldsymbol{H}^{n}$ but will use a more direct method to show that we have normlized the metrics correctly. See $\S 3.3$.

We will use the ball model for $\boldsymbol{H}^{N}$ and let $O$ denote the obvious origin in this model. Up to rotations about $O$ there is a canonical map $H: X \rightarrow \boldsymbol{H}^{N}$ which we call the Hadamard map. This is map is defined (up to rotations) by saying that $H$ is an isometry at $O$ and maps the geodesics through $O$ in $X$ to the geodesics through $O$ in $\boldsymbol{H}^{N}$. According to Hadamard's Theorem, the exponential map is a diffeomorphism in non-positive curvature, and this makes $H$ into a diffeomorphism. In the next chapter we will derive Equation 9, which is a fairly explicit formula for $H$. Even without the formula, however, we can say:

Lemma 2.5 $H$ is distance non-decreasing.

Proof: This is a well-known comparison theorem in Riemannian geometry. Here is a sketch: Say that a radial geodesic in either space is a geodesic through $O$. By definition $H$ is an isometry when restricted to each radial geodesic. $H$ maps the spheres about $O$ in $X$ to the spheres about $O$ in $\boldsymbol{H}^{N}$ and by the Gauss lemma (or just plain symmetry in the cases of interest to us) these spheres are orthogonal to the radial geodesics. Finally, the restriction of $H$ to each sphere is distance non-decreasing because the Jacobi fields diverge faster when the curvature is more negative. Putting these 3 ingredients together we see that $d H$ is distance non-decreasing on each tangent space of $X$.

Here is the comparison result:

Lemma 2.6 Let $W \subset X$. Then $W$ is a steering wheel in $X$ provided that $H(W)$ is a steering wheel in $\boldsymbol{H}^{N}$.

Proof: Let $B_{\infty} \subset X$ be a special horoball. We need to show that $B_{\infty}$ contains a point of $W$ in its interior. Let $B_{\infty}^{\prime}=H\left(B_{\infty}\right)$. If we knew that $B_{\infty}^{\prime}$ contains a 
special horosphere in $\boldsymbol{H}^{N}$ then we would conclude that $B_{\infty}^{\prime}$ contains a point of $H(W)$ in its interior. This would complete our proof.

$B_{\infty}$ is the geometric limit of unboundedly metric balls $\left\{B_{n}\right\}$. We can assume that $0 \in \partial B_{n}$ for all $n$. It suffices to show that $H\left(B_{n}\right)$ contains an unboundedly large metric ball $B_{n}^{\prime}$ such that $0 \in \partial B_{n}^{\prime}$. Let $x_{n}$ be the center of $B_{n}^{\prime}$. Let $r_{n}$ be the radius of $B_{n}^{\prime}$. Let $x_{n}^{\prime}=H\left(x_{n}\right)$. Note that the distance from $x_{n}^{\prime}$ to $O$ is the same as the distance from $x_{n}$ to $O$, namely $r_{n}$. Since $H$ is distance non-decreasing, we see that $H\left(B_{n}\right)$ contains the ball $B_{n}^{\prime}$ of hyperbolic radius $r_{n}$ about $x_{n}^{\prime}$. by construction $0 \in \partial B_{n}^{\prime}$. This completes our proof.

\subsection{Hyperbolic Steering Wheels}

Here we explain how to check the steering wheel condition for a finite subset $W=\left\{x_{1}, \ldots, x_{k}\right\} \subset \boldsymbol{H}^{N}$. Let $S_{1}$ denote the unit sphere. Define

$$
(W ; j)=\left\{y \in S_{1} \mid\left\|y-2 x_{j}\right\|<1\right\} .
$$

Here we are using the Euclidean metric.

Lemma 2.7 $W$ is a steering wheel if and only if $S_{1} \subset \bigcup(W ; j)$.

Proof: This is really a tautology. Let $S_{1 / 2}$ denote the sphere of radius $1 / 2$ about $O$. The special horoballs in our model are Euclidean balls having radius $1 / 2$ and centered on points of $S_{1 / 2}$. Let $\frac{1}{2} y$ be the center of such a special horoball $B$. Then $B$ contains $x_{j}$ in its interior if and only if $\left\|\frac{1}{2} y-x_{j}\right\|<1 / 2$, which is true if and only if $\left\|y-2 x_{j}\right\|<1$.

The spherical ball $(W ; j)$ has a radius which depends only on the Euclidean norm $\left\|x_{j}\right\|$ :

Lemma 2.8 Let $u_{j}=x_{j} /\left\|x_{j}\right\|$. Then $(W ; j)=S_{1} \cap B_{j}$ where $B_{j}$ is the ball of Euclidean radius $\rho_{j}=\sqrt{2-2\left\|x_{j}\right\|}$ centered at $u_{j}$.

Proof: Everything about this lemma follows from symmetry except the calculation of the radius $R$ of $B_{j}$. To compute $R$ note that $\partial(W ; j)$ is the intersection of the unit sphere about $2 x_{j}$ with the unit sphere about $O$. This intersection has the following description: Let $\Pi_{j}$ be the hyperplane perpendicular to $\overline{O x_{j}}$ 
and containing $x_{j}$. Then $\partial(W ; j)=\Pi_{j} \cap S_{1}$. Let $r$ denote the radius of $\partial(W ; j)$. From the Pythagorean theorem we get the two equations:

$$
r^{2}+\left(1-\left\|x_{j}\right\|\right)^{2}=R^{2} ; \quad r^{2}+\left\|x_{j}\right\|^{2}=1 .
$$

Hence $R^{2}=2-2\left\|x_{j}\right\|$

At this point we have an entirely Euclidean problem. We have a finite collection of balls $B_{1}, \ldots, B_{k}$ and we want to verify that these balls cover $S_{1}$. There are various algoriths one can use to decide this question. Here we will present a sufficient (but not always necessary) condition based on some crude properties of the convex hull $\operatorname{Hull}(U)$, where $U=\left\{u_{1}, \ldots, u_{k}\right\}$.

Let $x_{1}, \ldots, x_{k}$ be the elements of $H(W)$. Let $|W|=\max \left\|x_{j}\right\|$. Let $B_{1}, \ldots, B_{k}$ and $u_{1}, \ldots, u_{k}$ and $\rho_{1}, \ldots, \rho_{k}$ be as in Lemma 2.8 .

Lemma 2.9 (Covering Criterion) Suppose the ball of radius $|W|$ centered at the origin is contained in $\operatorname{Hull}(U)$. Then $S_{1} \subset B_{1} \cup \ldots \cup B_{n}$.

Now we sketch how we apply this criterion in practice. At first glance it appears that we would need to calculate $\operatorname{Hull}(U)$, a daunting feat in high dimensions. We will get around this problem with a trick:

- To show that $\operatorname{Hull}(U)$ contains the ball of radius $|W|$ centered at the origin, it suffices to show that $\operatorname{Hull}(U)$ contains the vertices of another polytope which contains this ball. In our example our auxilliary polytope will be a 9 dimensional cross polytope.

- To show that a particular vertex $v$ of the auxilliary polytope lies in $\operatorname{Hull}(U)$ we will simply exhibit it as a weighted average of the vertices of $U$. We find the weights by a rapidly converging iterative algorithm.

In $\S 4.5$ we will actually apply this method, so that the reader can see it in action.

\subsection{Proof of Lemma 2.9}

We will first establish some preliminary results about spherical geometry.

Say that the roundout of a set $A \subset \boldsymbol{R}^{N}-O$ is the radial projection of $A$ to $S_{1}$. Say that a $d$-dimensional geodesic spherical polytope in $S_{1}$ is the roundout of a $d$ dimensional Euclidean convex polytope whose vertices all lie in the interior 
of a hemisphere of $S_{1}$. A geodesic spherical polytope is bounded by a finite union of codimension 1 faces, all of which are geodesic spherical polytopes. A spherical ball on $S_{1}$ is a metric ball which is contained in a hemisphere. We define the chordal radius of a spherical ball to be the Euclidean distance from the boundary to the center of the ball on $S_{1}$.

Lemma 2.10 Let $B$ be a spherical geodesic ball of chordal radius $\rho$. Let $\Pi$ be a hyperplane through the origin. Let $B^{*}=\Pi \cap B$ and let $\rho^{*}$ be the chordal radius of $B^{*}$ in $S_{1}^{*}=S_{1} \cap \Pi$. Then $\rho^{*} \leq \rho$.

Proof: The chordal radius of $B$ is a monotone function of the Euclidean radius of $\partial B$. The function in question is independent of dimension, by symmetry. $S_{1}^{*}$ is an $N-1$ dimensional unit sphere and so we can compare the chordal radii of $B$ and $B^{*}$ by looking at the Euclidean radii of $\partial B$ and $\partial B^{*}$. Since $\partial B^{*}=\partial B \cap \Pi$ the Euclidean radius of $\partial B^{*}$ is at most that of $B$. Hence, the same comparison holds for the chordal radii.

Lemma 2.11 Suppose $P$ is a top dimensional geodesic spherical polytope whose vertices are contained in a spherical ball $B$ of radius $\rho$. Then $P \subset B$.

Proof: $P$ is the roundout of a convex polytope which lies in a hyperplane whose intersection with $S_{1}$ is separated from $O$ by the hyperplane containing $\partial B$. Hence $P \subset B$.

Lemma 2.12 Suppose $P$ is a top dimensional geodesic spherical polytope whose vertices are contained in a spherical ball $B$ of radius $\rho$. Then each point of $P$ is within $\rho$ of some vertex of $P$.

Proof: The case $N=2$ just involves arcs on the unit circle, and is pretty obvious. Now suppose that $N \geq 3$. Let $c \in S_{1}$ be the center of $B$. Suppose that we have some point $x \in P$ which is more than $\rho$ from all the vertices of $P$. We have $P \subset B$ by Lemma 2.11. Let $\gamma$ be the geodesic in $S_{1}$ which joins $c$ to $x$. The geodesic segment $\gamma \cap B$ must intersect $\partial P$ at some point $x^{*}$ such that $x$ lies between $c$ and $x^{*}$. Let $P^{*}$ be a codimension 1 face of $P$ which contains $x^{*}$. There is a hyperplane $\Pi$ through the origin such that $P^{*}=P \cap \Pi$. Let $B^{*}=B \cap \Pi$. Then $B^{*}$ contains the vertices of $P^{*}$ and has chordal radius at most $\rho$ by Lemma 
2.10. By induction there is some vertex $v$ of $P^{*}$ which is at most $\rho$ from $x^{*}$. But $v$ is also at most $\rho$ from $c$.

Let $B_{v}$ denote the spherical ball of chordal radius $\rho$ centered at $v$. Since $B_{v}$ is contained in a hemisphere, it is a locally convex subset of $S_{1}$. Hence $B_{v}$ contains the shorter of the two geodesic arcs joining $x^{*}$ to $c$. The geodesic arc of interest to us joins $x^{*}$ to $c$ and is a subarc of $\gamma \cap B$, an arc which is already shorter than a semicircle. Hence the geodesic arc of interest to us is the shorter of the two geodesic arcs joining $x^{*}$ to $c$. Hence $x \in B_{v}$, a contradiction.

Now we turn to the proof of the Covering Lemma. From the hypotheses in the Covering Criterion, $\operatorname{Hull}(U)$ contains $O$ in its interior. But this easily implies that $S_{1}$ is partitioned by the geodesic polytopes which are the roundouts of the faces of Hull $(U)$. Hence, to establish our Covering Criterion it suffices to prove that every point of the roundout $f$ of a face $f^{\prime}$ is within $\rho=\min \rho_{j}$ of some vertex of $f^{\prime}$.

The face $f^{\prime}$ is contained in a hyperplane $\Pi$ and the vertices of $f^{\prime}$ are contained in the sphere $\Pi \cap S_{1}$. Let $y \in S_{1}$ be the center of the spherical ball $B$ which is the smaller of the two components of $S_{1}-\Pi$. By hypotheses, the distance from $\Pi$ to $O$ is at least $|W|$. But, looking at the algebra done in Lemma 2.8, we see that the chordal radius of $B$ is at most $\sqrt{2-2|W|}=\rho$. Hence every vertex of $f^{\prime}$ is within $\rho$ of $y$. Lemma 2.12 now tells us that every point of $f$ is within $\rho$ of a vertex of $f^{\prime}$. This is what we had wanted to prove. 


\section{Some Useful Formulas}

\subsection{Metric Properties of $X$}

Let $X=S L_{n}(\boldsymbol{R}) / S O(n)$ be the symmetric space associated to $S L_{n}(\boldsymbol{R})$. We can identify $X$ with the space of $n \times n$ symmetric matrices having positive eigenvalues and determinant 1 . We have $\operatorname{dim}(X)=N$, where

$$
N=\frac{n(n+1)}{2}-1 \text {. }
$$

The origin $O$ of $X$ is naturally the identity matrix.

The tangent space $V=T_{O}(X)$ can be identified with the vector space of trace 0 symmetric matrices. This space has a natural inner product:

$$
\left\langle M_{1}, M_{2}\right\rangle=\operatorname{Tr}\left(\frac{1}{2} M_{1} M_{2}\right) .
$$

The factor of $1 / 2$ is a normalizing constant. We will discuss our normalization below in $\S 3.3$.

The action of $S L_{n}(\boldsymbol{R})$ on $X$ is given by

$$
M(S)=M S M^{t} .
$$

Here $M^{t}$ is the transpose of $M$. The stabilizer of $O$ is $S O(n)$. If $M \in S O(n)$ we have $M^{t}=M^{-1}$. Hence $S O(n)$ acts on $V$ by conjugation and the inner product in Equation 4 is $S O(n)$-invariant. We get a Riemannian metric on $X$ by transporting our inner product on $V$ to all of the tangent bundle, using the action of $S L_{n}(\boldsymbol{R})$. As usual, the (global) metric on $X$ is defined in terms of the lengths of the geodesics with respect to the Riemannian metric.

The exponential map exp : $V \rightarrow X$ is given by:

$$
\exp (M)=\sum_{k=0}^{\infty} \frac{M^{k}}{k !}
$$

For any $x \in X$ we can write $x=M^{-1} D M$ Where $M \in S O(n)$ and $D$ is a diagonal matrix with positive entries $\lambda_{1}, \ldots, \lambda_{n}$. Let $\log D$ be the diagonal matrix with entries $\log \left(\lambda_{1}\right), \ldots, \log \left(\lambda_{n}\right)$. Setting $\log x=M^{-1}(\log D) M$ we have $\exp (\log x)=$ $x$.. We have the distance formula

$$
d(x, O)=\|\log x\|=\|\log D\|=\left[\sum_{i=1}^{n} \frac{1}{2} \log ^{2}\left(\lambda_{i}\right)\right]^{1 / 2}
$$

This is all we need to know about the metric on $X$. 


\subsection{A Formula for the Hadamard Map}

Here we will consider $\boldsymbol{H}^{N}$, where $N=\operatorname{dim}(X)$ and $X$ is the symmetric space considered above. We have already equipped $V=T_{O}(X)$ with an inner product and so we may isometrically identify $V$ with $\boldsymbol{R}^{N}$. Our model for $\boldsymbol{H}^{N}$ is the open unit ball in $V$. If $x \in \boldsymbol{H}^{N}$ is any point we let $\|x\|<1$ denote the Euclidean norm of $x$. It turns out that the hyperbolic distance from $O$ to $x$ is given by

$$
d(O, x)=\log \frac{1+\|x\|}{1-\|x\|} .
$$

See $[\mathbf{B}$, p. 38].

Now we give the formula for the Hadamard map. Let $x \in X-O$. We write $x=M D M^{-1}$ as above. Then

$$
\begin{gathered}
H(x)=\tanh (d / 2) u ; \\
u=\frac{\log x}{\|\log x\|} ; \quad d=\|\log x\|=\sqrt{\operatorname{Tr}\left((\log x)^{2} / 2\right)} .
\end{gathered}
$$

To see why this works, note that $u$ is a unit vector in $V$ which points in the same direction $\operatorname{as} \log x$ and $d=d(0, x)$. Hence $\exp (d u)=x$. By construction $H(x)$ is a scalar multiple of $\log x$ in $V$ and the hyperbolic distance from $O$ to $H(x)$ is exactly $d$ because

$$
d^{\prime}=\tanh \frac{d}{2} \quad \Longrightarrow \quad \log \frac{1+d^{\prime}}{1-d^{\prime}}=d
$$

There is a very nice isometric embedding of $V$ into a subspace of $\boldsymbol{R}^{N+1}$. Here $\boldsymbol{R}^{N+1}$ is given the standard metric. We simply string out the upper triangular part of $V$ in a certain order, starting down the main diagonal and working away from the main diagonal. To get the embedding to be an isometric one, we need to multiply all the diagonal entries by $1 / \sqrt{2}$. (See the computer code in $\S 3.4$ for the exact recipe.) The image of this embedding is the subspace $\boldsymbol{R}_{0}^{n} \times \boldsymbol{R}^{N-n+1}$ where $\boldsymbol{R}_{0}^{n} \subset \boldsymbol{R}^{n}$ consists of all the vectors whose coordinates sum to 0 . Given this embedding, the norm $\|\cdot\|$ refers equally well to the standard norm on $\boldsymbol{R}^{N}$ and the one on $V$ which comes from Equation 4.

\subsection{Double Checking the Normalization}

In this section we check that the sectional curvature of $X$ is bounded below by the sectional curvature of $\boldsymbol{H}^{N}$. Rather than compute these curvatures directly 
we will show, for $n=2$, that $H: X \rightarrow \boldsymbol{H}^{2}$ is an isometry, as it should be. By symmetry, $H$ is an isometry when restricted to certain totally geodesic copies of $\boldsymbol{H}^{2}$ embedded in the general $X$. This is a sufficient check.

To show that $H$ is an isometry in the case $n=2$ it suffices to exhibit a single triangle, $\Delta \subset X$, with one vertex the origin, such that $\Delta$ and $H(\Delta)$ are isometric. We choose the vertices of $\Delta$ to be

$$
x_{1}=\left[\begin{array}{cc}
1 & 0 \\
0 & 1
\end{array}\right] ; \quad x_{2}=\left[\begin{array}{cc}
2 & 0 \\
0 & 1 / 2
\end{array}\right] ; \quad x_{3}=\left[\begin{array}{cc}
5 / 4 & -3 / 4 \\
-3 / 4 & 5 / 4
\end{array}\right]
$$

Note that $x_{3}=R x_{2} R^{t}$ where

$$
R=\left[\begin{array}{cc}
\cos (\pi / 4) & \sin (\pi / 4) \\
-\sin (\pi / 4) & \cos (\pi / 4)
\end{array}\right] .
$$

$R$ acts on $X$ as a rotation by $\pi / 2$ so that $\Delta$ is a right-anged isosceles triangle.

First we compute distances in $X$. For later use we point out that, for $n=2$, we have $d_{X}(O, S)=\log (\lambda)$, where $\lambda$ is the larger of the two eigenvalues of the symmetric matrix $S$. Hence $d_{X}\left(x_{1}, x_{2}\right)=\log (2)$. By symmetry, $d_{X}\left(x_{1}, x_{3}\right)=$ $\log (2)$.

To compute $d_{X}\left(x_{2}, x_{3}\right)$ we note that $x_{1}=M x_{2} M^{t}$ where

$$
M=x_{2}^{-1 / 2}=\left[\begin{array}{cc}
\sqrt{1} / 2 & 0 \\
0 & \sqrt{2}
\end{array}\right]
$$

Hence

$$
d_{X}\left(x_{2}, x_{3}\right)=d_{X}\left(M x_{2} M^{t}, M x_{3} M^{t}\right)=d_{X}\left(x_{1}, M x_{3} M^{t}\right),
$$

where

$$
M x_{3} M^{t}=\left[\begin{array}{cc}
5 / 8 & -3 / 4 \\
-3 / 4 & 5 / 2
\end{array}\right] .
$$

This last matrix has eigenvalues

$$
\frac{1}{16}(25 \pm 3 \sqrt{41})
$$

Hence,

$$
\left.d_{X}\left(x_{2}, x_{3}\right)=\log \left(\frac{1}{16}(25+3 \sqrt{41})\right)\right) .
$$

Now we compute distances in $\boldsymbol{H}^{2}$. Let $\delta$ denote the hyperbolic metric. In the notation from $\S 3.2$ (with subscripts added) we have

$$
d_{2}=\left\|\log x_{2}\right\|=\log (2) ; \quad u_{2}=\frac{\log x_{2}}{d}=\left[\begin{array}{cc}
1 & 0 \\
0 & -1
\end{array}\right]
$$




$$
\begin{gathered}
d_{2}^{\prime}=\tanh \left(d_{2} / 2\right)=1 / 3 ; \quad H\left(x_{2}\right)=d_{2}^{\prime} u_{2}=\left[\begin{array}{cc}
1 / 3 & 0 \\
0 & -1 / 3
\end{array}\right] ; \\
H\left(x_{3}\right)=R H\left(x_{2}\right) R^{-1}=\left[\begin{array}{cc}
0 & -1 / 3 \\
-1 / 3 & 0
\end{array}\right] .
\end{gathered}
$$

For the last equation we are using the fact that $R^{t}=R^{-1}$.

The identification

$$
M=\left[\begin{array}{cc}
a & b \\
b & -a
\end{array}\right] \rightarrow a-b i
$$

is an isometry from $T_{O}(X)$ to $\boldsymbol{C}$ because $\|M\|=\sqrt{a^{2}+b^{2}}$. This identification yields

$$
H\left(x_{1}\right) \rightarrow 0 ; \quad H\left(x_{2}\right) \rightarrow 1 / 3 ; \quad H\left(x_{3}\right) \rightarrow i / 3 .
$$

We denote these points respectively by $y_{1}, y_{2}, y_{3}$. From Equation 8 we get $\delta\left(y_{1}, y_{2}\right)=$ $\delta\left(y_{1}, y_{3}\right)=\log (2)$. To compute $\delta\left(y_{2}, y_{3}\right)$ we introduce the linear fractional transformation

$$
T(z)=\frac{z-1 / 3}{1-z / 3} .
$$

Then $T$ is a hyperbolic isometry such that $T(1 / 3)=0$. We have

$$
T(i / 3)=\frac{-15}{41}+\frac{12}{41} i ; \quad\|T(i / 3)\|=3 / \sqrt{41} .
$$

We have

$$
\delta\left(y_{2}, y_{3}\right)=\delta(0, T(i / 3))=\log \frac{1+3 / \sqrt{41}}{1-3 / \sqrt{4} 1}=\log \left(\frac{1}{16}(25+3 \sqrt{4} 1)\right)=d_{X}\left(x_{2}, x_{3}\right) .
$$

This completes our verification.

Remark: As an extra check, we note that $\cosh \left(d_{X}\left(x_{2}, x_{3}\right)\right)=(5 / 4)^{2}$ and $\cosh \left(d_{X}\left(x_{1}, x_{2}\right)\right)=5 / 4$, so that our distances satisfy the Hyperbolic Pythagorean Theorem for right-angled hyperbolic triangles. See [B, p 148].

\subsection{Taylor Series Approximation}

Here we give some approximations to the Hadamard map. These approximations output a rational vector given a rational matrix. (Though the final injection into $\boldsymbol{R}^{N}$ multiplies some of the coordinates by a factor of $\sqrt{2}$.)

Let $x \in X$. Let $m=x-I$ and $\lambda(x)=\sup \left|m_{i j}\right|$. As long as $\lambda<1$ we have

$$
\log x=\lim _{k \rightarrow \infty} L_{k}(x) ; \quad L_{k}(x)=\sum_{j=1}^{k}(-1)^{j+1} \frac{m^{j}}{j} .
$$


This can be proved by a series argument, as in the case of 1 real variable. Here are the approximations to the Hadamard map from Equation 9.

$$
\begin{gathered}
H_{k}(x)=\frac{1}{2} L_{k}(x)=\left(d_{k} / 2\right) u_{k} ; \\
u_{k}=\frac{L_{k}(x)}{\left\|L_{k}(x)\right\|} ; \quad d_{k}=\left\|L_{k}(x)\right\|=\sqrt{\operatorname{Tr}\left(L_{k}(x)^{2} / 2\right)}
\end{gathered}
$$

Lemma 3.1 Let $\lambda, u, d, u_{k}, d_{k}$ be as above. Assume that $\lambda<1$. We have

$$
\left\|u-u_{k}\right\| \leq \frac{\sqrt{2} E}{\left\|L_{k}(x)\right\|} ; \quad\|H(x)\| \leq\left\|H_{k}(x)\right\|+\frac{E}{\sqrt{8}} ; \quad E=\frac{N \lambda^{k+1}}{(k+1)(1-\lambda)} ;
$$

Proof: The entries in the matrix $\mu=\log x-L_{k}(x)$ all have absolute value at most

$$
\frac{\lambda^{k+1}}{k+1}+\frac{\lambda^{k+2}}{k+2}+\frac{\lambda^{k+3}}{k+3} \ldots \leq \frac{\lambda^{k+1}}{k+1}\left(1+\lambda+\lambda^{2}+\ldots\right)=\frac{\lambda^{k+1}}{(k+1)(1-\lambda)} .
$$

But then

$$
\left\|L_{k}(x)-\log x\right\|=\sqrt{\left|\operatorname{Tr}\left(\mu^{2} / 2\right)\right|} \leq E / \sqrt{2}
$$

There is a unique positive scalar multiple $L_{k}^{*}(x)$ of $u$ which has the property that $\left\|L_{k}^{*}(x)\right\|=\left\|L_{k}(x)\right\|$. From elementary geometry we have

$$
\left\|L_{k}(x)-L_{k}^{*}(x)\right\| \leq 2\left\|L_{k}(x)-\log x\right\| \leq \sqrt{2} E .
$$

Scaling $L_{k}(x)$ and $L_{k}^{*}(x)$ by $\left\|L_{k}(x)\right\|^{-1}$ we obtain $u_{k}$ and $u$ respectively. Our first estimate thus follows immediately from Equation 12. Our second estimate follows from Equation 11, from the triangle inequality, and from the fact that $\tanh (|t|) \leq|t|:$

$$
\tanh \frac{\|\log x\|}{2} \leq \frac{\|\log x\|}{2} \leq \frac{\left\|L_{k}(x)\right\|}{2}+\frac{E}{\sqrt{8}} .
$$

Remark: In $\S 4$ we will have

$$
N=10 ; \quad k=3 ; \quad \lambda<\frac{1}{15} ; \quad\left\|L_{3}(x)\right\| \in\left(\frac{1}{20}, \frac{1}{11}\right) .
$$

This leads to

$$
\left\|u-u_{3}\right\| \leq \frac{1}{1000} ; \quad\|H(x)\|<\left\|H_{3}(x)\right\|+\frac{1}{10000}<\frac{1}{20} .
$$




\subsection{Mathematica Code}

Here is a Mathematica version of our Hadamard map, in the case $n=4$. This version computes things numerically starting with 100 digits of accuracy. Presumably the final answer is slightly less accurate. The interested reader can treak the code in an obvious way to change the degree of accuracy.

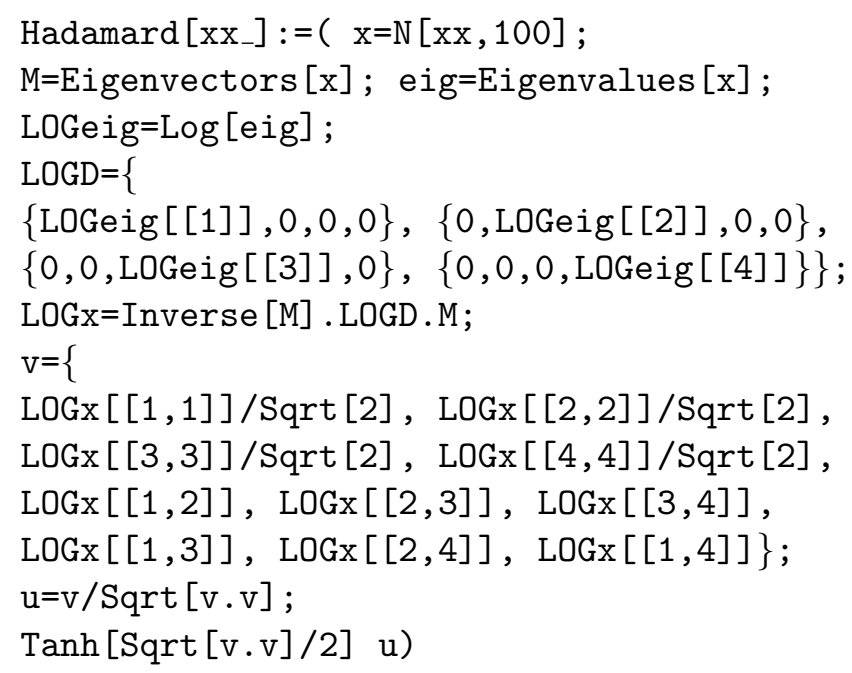

We don't actually use the above version in our proof. Rather, we use the $k=3$ approximation to the Hadamard map, together with the approximation results proved above. The reader interested in using the above version for rigorous purposes should have little difficulty getting it to work in Mathematica with interval arithmetic. Mathematica has built-in support for this.

As we just said, we shall work with the $k=3$ approximation to the Hadamard map. The code for this approximation is much simpler, and we display it for the case of interest to us, $n=4$.

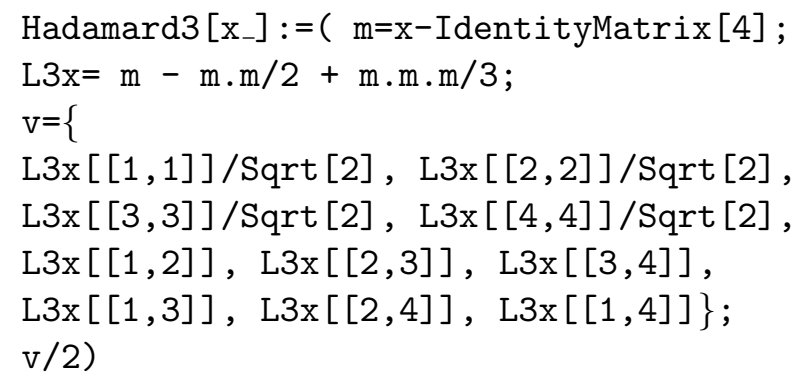




\section{Barycentric Subdivision}

\subsection{Basic Definitions}

Here we generalize the setup in $[\mathbf{S}]$. Let $S_{n+1}$ denote the permutation group of the symbols $\{0, \ldots, n\}$. Let $\Delta$ be the convex hull of the $n+1$ points $v_{0}, \ldots, v_{n}$ in $\boldsymbol{R}^{n}$. Given

$$
\sigma=\left(i_{0}, \ldots, i_{n}\right) \in S_{n+1}
$$

we let $c_{k}$ denote the center of mass of the points $v_{i_{0}}, \ldots, v_{i_{k}}$. Concretely,

$$
c_{k}=\frac{1}{k+1} \sum_{j=0}^{k} v_{i_{j}} .
$$

Let $\Delta_{\sigma}$ denote the convex hull of the points $c_{0}, \ldots, c_{n}$. The union $\bigcup_{\sigma} \Delta_{\sigma}$ is precisely the barycentric subdivision of $\Delta$.

We begin our process by taking our initial simplex to be the convex hull of the points $e_{0}, e_{1}, \ldots, e_{n}$. Here $e_{0}=O$ and $e_{1}, \ldots, e_{n}$ are the standard basis vectors in $\boldsymbol{R}^{n}$.

Remark: It might seem more natural to take the initial simplex to be the regular simplex. However, our choice leads to much simpler formulas.

Given $\sigma \in S_{n+1}$ we let $A_{\sigma}$ denote the unique affine map such that $A_{\sigma}\left(e_{k}\right)=c_{k}$ for all $k$. (Here $c_{0}, \ldots, c_{n}$ depend on $\sigma$, as above.) Next, we define

$$
T_{\sigma}= \pm \frac{L_{\sigma}}{\left|\operatorname{det}\left(L_{\sigma}\right)\right|^{1 / n}}
$$

where $L_{\sigma}$ is the linear part of $A_{\sigma}$. As in $\S 1$, the sign is chosen to make the determinant positive. Note that $A_{\sigma}=L_{\sigma}+c_{0}$. Hence

$$
L_{\sigma}=\left[\begin{array}{lll}
c_{1}-c_{0} & \ldots & c_{n}-c_{0}
\end{array}\right]
$$

Thus $T_{\sigma}$ is quite easy to compute.

We order the elements of $S_{n+1}$ lexicographically and let $T_{j}=T_{\sigma_{j}}$, where $\sigma_{j}$ is the $j$ th permutation in our ordering. Then $\Omega_{n}$ is generated by the elements $T_{1}, \ldots, T_{(n+1) !}$ together with the affine symmetries of our initial simplex. (Actually, $\Omega_{n}$ is generated by just $T_{1}$ and the affine symmetries.) In particular, $\Omega_{n}$ contains all the permutation matrices (up to sign). These permutation matrices will come in quite handy during our proof. 


\subsection{Mathematica Version}

Here is some Mathematica code which computes the $T_{j}$ for $n=4$. The code can easily be modified to work for other values of $n$-e.g. $n=2,3$.

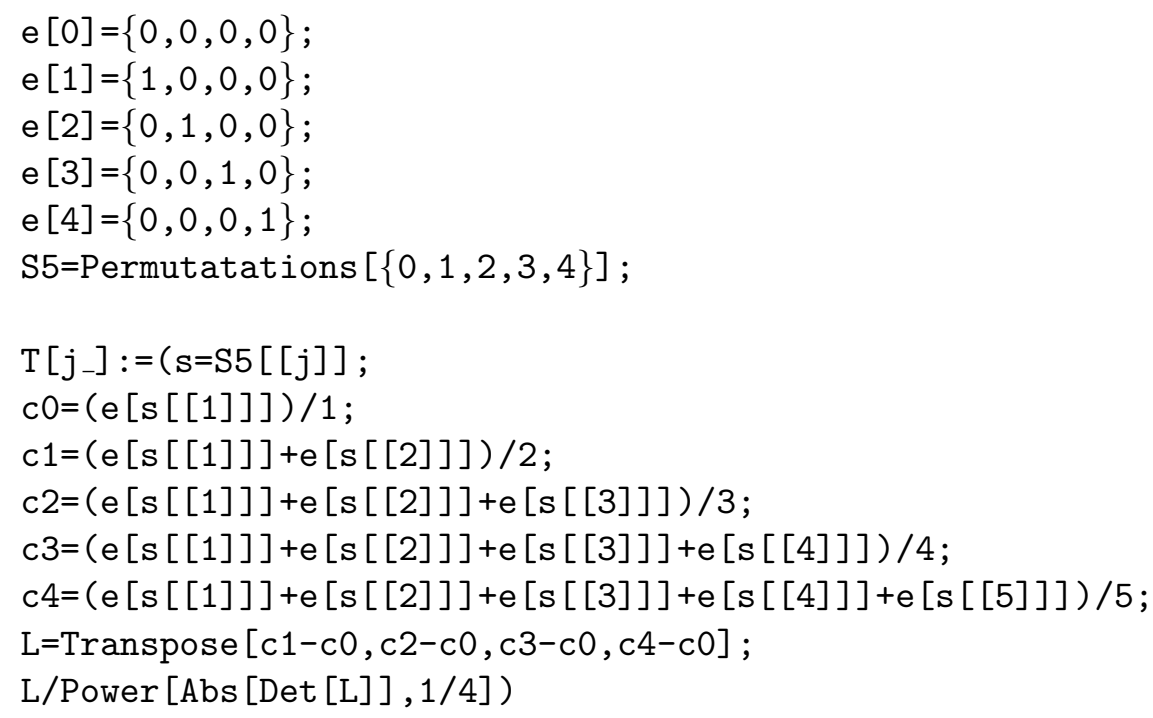

Here are the examples of infinite order elliptics promised in the introduction. We introduce the notation $\langle a b c \ldots\rangle=T_{a} T_{b} T_{c} \ldots$

In the 2 dimensional case we compute that

$$
\langle 14\rangle=\left[\begin{array}{cc}
-1 / 2 & -4 / 3 \\
1 & 2 / 3
\end{array}\right]
$$

This matrix is easily seen to be infinite order elliptic. In the 3 dimensional case we compute that

$$
\langle 161619\rangle=-\left[\begin{array}{ccc}
3 / 4 & 5 / 3 & 43 / 24 \\
-9 / 4 & -7 / 3 & -65 / 24 \\
9 / 4 & 5 / 3 & 37 / 24
\end{array}\right]
$$

This matrix has eigenvalues

$$
1 ; \quad \frac{-23 \pm 5 i \sqrt{71}}{48}
$$

and hence is infinite order elliptic. 


\subsection{The Steering Wheel}

For $n=4$ the space $X$ has dimension $N=9$. Logically speaking, it doesn't matter how we found our steering wheel for $\Omega_{4}$. We just have to verify that it works using the Covering Criterion from $\S 3$. However, it certainly seems worthwhile to explain how we found it.

By construction we have

$$
T_{a}= \pm P_{a} T_{1} ; \quad a \in[1,24] .
$$

Here $P_{a}$ is the $a$ th permutation matrix. These matrices are ordered lexicographically, as the permutations on which they are based.

As in the case $k=1$ of Equation 10 we define

$$
d_{1}(A)=\sqrt{\operatorname{Tr}\left(\left(A A^{t}-I\right)\left(A A^{t}-I\right)\right) / 2} .
$$

Step 1: $A_{1}, \ldots, A_{40}$ which minimize the quantity

$$
d_{1}(\langle 1 a b c\rangle) \quad a, b, c, d \in[1,120] .
$$

We like these matrices because they have rational entries.

Step 2: We find matrices $B_{1}, \ldots, B_{40}$ which minimize the quantity

$$
d_{1}\left(P_{1} A_{a} P_{b} A_{c} P_{d} A_{e}\right) ; \quad a, c, e \in[1,40] ; \quad b, d \in[1,24]
$$

Step 3: We find the matrices $C_{1}, C_{2}, C_{3}, C_{4}, C_{5}$ which minimize the quantity

$$
d_{1}\left(P_{1} B_{a} P_{b} B_{c} P_{d} B_{e}\right) ; \quad a, c, e \in[1,40] ; \quad b, d \in[1,24]
$$

Remark: Step 2 is really the induction step for an iterative algorithm which presumably would produce a sequence of elements in $\Omega_{4}$ converging to the identity. However, the whole point of this paper is that we can stop after a finite amount of computation and prove our density result. Incidentally, there is nothing magic about the number 40. We just found that it worked well for us in Steps 1 and 2.

Our steering wheel $W$ is made from the 120 elements:

$$
\pm P_{a} C_{b} ; \quad a \in[1,24] ; \quad b \in[1,5] .
$$


Here are the equations for the $C$ matrices:

$$
\begin{aligned}
& C_{1}=\left\langle\begin{array}{cccccccccccc}
1 & 19 & 108 & 63 & 12 & 52 & 96 & 57 & 16 & 52 & 49 & 95 \\
6 & 54 & 70 & 87 & 19 & 25 & 84 & 87 & 2 & 99 & 60 & 64 \\
18 & 82 & 108 & 64 & 3 & 43 & 108 & 57 & 11 & 82 & 108 & 64
\end{array}\right\rangle \\
& C_{2}=\left\langle\begin{array}{cccccccccccc}
1 & 54 & 70 & 87 & 18 & 108 & 46 & 64 & 4 & 58 & 78 & 89 \\
9 & 52 & 49 & 95 & 7 & 52 & 49 & 95 & 22 & 79 & 60 & 64 \\
13 & 53 & 84 & 63 & 18 & 43 & 108 & 63 & 17 & 52 & 49 & 95
\end{array}\right\rangle \\
& C_{3}=\left\langle\begin{array}{cccccccccccc}
1 & 54 & 70 & 87 & 18 & 108 & 46 & 64 & 4 & 58 & 78 & 89 \\
3 & 8 & 108 & 63 & 12 & 19 & 108 & 63 & 11 & 58 & 78 & 89 \\
3 & 30 & 70 & 87 & 9 & 53 & 84 & 63 & 23 & 108 & 46 & 64
\end{array}\right\rangle \\
& C_{3}=\left\langle\begin{array}{cccccccccccc}
1 & 6 & 58 & 63 & 22 & 53 & 60 & 64 & 11 & 8 & 108 & 57 \\
20 & 19 & 108 & 63 & 12 & 52 & 96 & 57 & 16 & 52 & 49 & 95 \\
8 & 58 & 78 & 89 & 20 & 111 & 60 & 64 & 4 & 108 & 46 & 64
\end{array}\right\rangle \\
& C_{3}=\left\langle\begin{array}{cccccccccccc}
1 & 13 & 108 & 63 & 23 & 8 & 108 & 63 & 11 & 43 & 108 & 57 \\
9 & 54 & 70 & 87 & 18 & 108 & 46 & 64 & 4 & 58 & 78 & 89 \\
15 & 80 & 108 & 64 & 6 & 53 & 84 & 63 & 9 & 54 & 70 & 87
\end{array}\right\rangle
\end{aligned}
$$

These arrays are meant to be read like a page of text: left to right and then down.

\subsection{The Approximate Hadamard Image}

Our steering wheel $W$ consists of the 120 symmetric $4 \times 4$ matrices of the form $M M^{t}$ where $M$ is one of the 120 matrices $P_{a} C_{b}$ listed above. To find the image $H(W) \subset \boldsymbol{R}^{10}$ we would simply apply the Hadamard map to each of the 5 matrices $C_{j} C_{j}^{t}$ and then use the symmetry coming from the permutation matrices. To understand the action of the matrix $P_{a}$ on $\boldsymbol{R}^{10}$ we write

$$
\boldsymbol{R}^{10}=\boldsymbol{R}^{\left(\begin{array}{l}
4 \\
1
\end{array}\right)} \times \boldsymbol{R}^{\left(\begin{array}{l}
4 \\
2
\end{array}\right)}
$$

The coordinates in the first factor are indexed by the 1-element subsets of $\{1,2,3,4\}$ and the coordinates in the second factor are indexed by 2 element subsets of 
$\{1,2,3,4\}$. A permutation then permutes the first 4 coordinates and also permutes the 2-element subsets indexing the second coordinates. This is the action. The image of the Hadamard map is contained in the subspace $V=\boldsymbol{R}_{0}^{4} \times \boldsymbol{R}^{6}$ where $\boldsymbol{R}_{0}^{4}=\left\{x_{1}+x_{2}+x_{3}+x_{4}=0\right\}$.

To make our calculation more transparent we use the approximation $H_{3}$ from $\S 3.3$, rather than the Hadamard map $H$, and then estimate the difference. Here we list the first 6 decimal places of the vectors

$$
H_{3}\left(C_{j} C_{j}^{t}\right) /\left\|H_{3}\left(C_{j} C_{j}^{t}\right)\right\| ; \quad j=1,2,3,4,5
$$

$\left[\begin{array}{c}0.395038 \\ 0.102796 \\ 0.0115207 \\ -0.509306 \\ -0.23079 \\ -0.158615 \\ -0.404498 \\ -0.335099 \\ 0.263012 \\ -0.387744\end{array}\right]\left[\begin{array}{c}0.343731 \\ 0.0323703 \\ -0.209797 \\ -0.166216 \\ 0.36621 \\ 0.485628 \\ 0.582669 \\ 0.287643 \\ -0.12911 \\ 0.0174091\end{array}\right]\left[\begin{array}{c}-0.127014 \\ 0.136948 \\ 0.48439 \\ -0.494167 \\ -0.569276 \\ 0.230596 \\ 0.145616 \\ 0.000420745 \\ 0.291821 \\ -0.0516275\end{array}\right]\left[\begin{array}{c}0.193941 \\ -0.157682 \\ 0.123853 \\ -0.160004 \\ 0.199935 \\ -0.662376 \\ -0.45887 \\ -0.129582 \\ -0.435437 \\ 0.0301193\end{array}\right]\left[\begin{array}{c}0.401339 \\ -0.259734 \\ 0.295396 \\ -0.436882 \\ -0.517536 \\ 0.263641 \\ 0.14662 \\ 0.178276 \\ -0.233664, \\ -0.219345\end{array}\right]$

We record the norms $\left\|H_{3}\left(C_{j} C_{j}^{t}\right)\right\|$ as

$\begin{array}{lllll}.027255 \ldots & .03185 \ldots & .044356 \ldots & .033822 \ldots & .0388857 \ldots\end{array}$

We record the vales $\lambda\left(C_{j} C_{j}^{t}\right)$ as

$$
\begin{array}{llllll}
.0379474 \ldots & .0364044 \ldots & .062955 \ldots & .0443729 \ldots & .046559
\end{array}
$$

From here we see that Equation 13 holds for $C_{1}, \ldots, C_{5}$. Hence $\|H(x)\|<1 / 20$ by Equation 14. Hence $H(W)$ is contained in the ball of Euclidean radius 1/20 about $O$.

\subsection{Estimating the Convex Hull}

To show that $H(W)$ is a steering wheel, it suffices to show that $\mathrm{Hull}(U)$ contains the ball of radius $1 / 20$ about $O$. Let $U_{3}$ denote the approximation to $U$ obtained when we use $H_{3}$ instead of $H$. From Equation 14, each point of $U$ is within $\frac{1}{1000}$ of a point of $U_{3}$ and vice versa. So, any point in $\operatorname{Hull}(U)$ is within $\frac{1}{1000}$ of a point of $\operatorname{Hull}\left(U_{3}\right)$, and vice versa. Hence, it suffices to show that $\operatorname{Hull}\left(U_{3}\right)$ contains the ball of radius $3 / 40>1 / 20+1 / 1000$ about $O$. 
Let $Z$ be the convex hull of the union of the $24 \times 3$ points $P_{a}\left(z_{b}\right)$ where

$$
z_{1}=.3(0, \ldots, 0,1) ; \quad z_{2}=-z_{1} ; \quad z_{3}=.15(1,1,-1,-1,0, \ldots, 0) .
$$

Then $Z$ is centrally symmetric and each pair of antipodal vertices is orthogonal to every other pair. Hence $Z$ is isometric to the 9 dimensional cross-polytope whose vertices are $3 / 10$ from the $O$. From here it is easy to see that $Z$ contains the ball of radius $1 / 10$ about $O$ in $\boldsymbol{R}_{0}^{4} \times \boldsymbol{R}^{6}$. We will try to show that $Z \subset \operatorname{Hull}\left(U_{3}\right)$. By symmetry, it suffices to show $z_{j} \in \operatorname{Hull}\left(U_{3}\right)$ for $j=1,2,3$. We perform the following iterative algorithm for each $z=z_{j}$ :

1. Let $\zeta_{0}=O$. Then $\zeta_{0} \in \operatorname{Hull}\left(U_{3}\right)$ by symmetry.

2. Assuming that $\zeta_{0}, \ldots, \zeta_{k}$ have been constructed let $\delta_{k}=\left\|z-\zeta_{k}\right\|$.

3. Let $\zeta_{k+1}$ be the point which minimizes the distance from $z$ to

$$
\left(1-\lambda \delta_{k}\right) \zeta_{k}+\lambda \delta_{k} u_{j} ; \quad j=1, \ldots, 120 .
$$

Here $u_{j} \in U_{3}$. (Geometrically, we move $\zeta_{k}$ a little bit of the way towards each of the points in $U_{3}$ and see which perturbation brings us closer to $z$.) As long as $\lambda<1 / 2$ we have $\zeta_{k} \in \operatorname{Hull}\left(U_{3}\right)$ for all $k$.

Along the way our algorithm produces weights $\left\{\omega_{k j}\right\}$ such that

$$
\zeta_{k}=\sum \omega_{k j} u_{j} ; \quad \sum \omega_{k j} \leq 1 ; \quad \omega_{k j} \geq 0 .
$$

\section{Remarks:}

(i) The value of $\lambda$ affects the convergence of the algorithm. We use $\lambda=1 / 8$.

(ii) If $U_{3}$ were a general collection of points in $S_{1}$ then we would have to exhibit the equation $z_{j}=\sum \omega_{i} u_{i}$ where $\sum \omega_{i}=1$ to show that $z_{j} \in \operatorname{Hull}\left(U_{3}\right)$. Here $\left\{\omega_{j}\right\}$ are the non-negative weights. However, in our case we know that $O \in \operatorname{Hull}\left(U_{3}\right)$ and so it is enough to take $\sum \omega_{j} \leq 1$.

We run our algorithm in C. We first run our algorithm for about 1000 iterations to get a rough sense of the weights. Then, to get a cleaner final answer, we eliminate any vertex whose weight is less than .0001 and run the algorithm again for 2000 iterations. (In the second iteration we really only perform Step 3 above for a small fraction of the 120 points. This is why most of the weights in our final answer are 0.) When we are done we have our list of weights. When we take the weighted average of our vectors, with the generated weights, we get a point $z_{j}^{\prime}$ which is within $10^{-12}$ of the point $z_{j}$. This calculation, which is accurate 
up to about 16 decimal places, shows that there is a polytope $Z^{\prime}$ is contained within $\operatorname{Hull}\left(U_{3}^{\prime}\right)$ where each point of $Z^{\prime}$ is easily within $10^{-12}$ of $Z$ and each point of $U_{3}^{\prime}$ is easily within $10^{-12}$ of $U_{3}$. This is enough information to see that, say, $.999 Z \subset \operatorname{Hull}\left(U_{3}\right)$, and $.999 Z$ contains the ball of radius $3 / 40$ centered at the origin. This completes our proof that $W$ is a steering wheel for $\Omega_{4}$.

For the record, we list the nonzero weights we get from our algorithm, up to 6 decimal places. In our listings, the coefficient $(a, b)$ stands for $P_{a}\left(H_{3}\left(C_{b} C_{b}^{t}\right)\right)$. Here are the listings for $z_{1}, z_{2}, z_{3}$ respectively:

$\begin{array}{cccc}(12,1): 0.000834 & (18,1): 0.002802 & (8,2): 0.006935 & (9,2): 0.081499 \\ (10,2): 0.096916 & (11,2): 0.022494 & (14,2): 0.006094 & (15,2): 0.079902 \\ (16,2): 0.080828 & (17,2): 0.019432 & (8,3): 0.022283 & (11,3): 0.007182 \\ (14,3): 0.007834 & (17,3): 0.007487 & (5,4): 0.045395 & (6,4): 0.065154 \\ (19,4): 0.038470 & (20,4): 0.039940 & (2,5): 0.029845 & (4,5): 0.057037 \\ (21,5): 0.028191 & (23,5): 0.034357 & & \\ & & & \\ & & & \\ (5,1): 0.001296 & (6,1): 0.012831 & (9,1): 0.012822 & (10,1): 0.017746 \\ (15,1): 0.004652 & (16,1): 0.010315 & (19,1): 0.010096 & (20,1): 0.024886 \\ (5,3): 0.074393 & (6,3): 0.103529 & (9,3): 0.008950 & (10,3): 0.006996 \\ (15,3): 0.018158 & (16,3): 0.003028 & (19,3): 0.092300 & (20,3): 0.055322 \\ (8,4): 0.037383 & (11,4): 0.030937 & (14,4): 0.025673 & (17,4): 0.042379 \\ & & & \\ & & & \\ (1,1): 0.008859 & (2,1): 0.013550 & (5,1): 0.017547 & (8,1): 0.002958 \\ (4,2): 0.013268 & (6,2): 0.033421 & (7,2): 0.020130 & (10,2): 0.037629 \\ (12,2): 0.027642 & (1,3): 0.000898 & (2,3): 0.002497 & (7,3): 0.010825 \\ (8,3): 0.005351 & (13,3): 0.000294 & (15,3): 0.008496 & (19,3): 0.010535 \\ (1,5): 0.010874 & (2,5): 0.038034 & (3,5): 0.104569 & (5,5): 0.052545 \\ (7,5): 0.034533 & (8,5): 0.011312 & (9,5): 0.108714 & (11,5): 0.060276\end{array}$




\section{Proof of Lemma 1.1}

\subsection{The Key Formulas}

Define the commutator $[R, S]=R S R^{-1} S^{-1}$ as usual. Using the notation from $\S 4.1$ we define

$$
A=\left[T_{1}, T_{2}\right]^{2} ; \quad B=\left[\left[T_{1}, T_{2}\right],\left[T_{1}, T_{7}\right]\right] .
$$

Note that $\left[T_{i}, T_{j}\right]=\left[L_{i}, L_{j}\right]$. We have

$$
L_{1}=\left[\begin{array}{ccccc}
\frac{1}{2} & \frac{1}{3} & \frac{1}{4} & \ldots & \frac{1}{n+1} \\
0 & \frac{1}{3} & \frac{1}{4} & \ldots & \frac{1}{n+1} \\
0 & 0 & \frac{1}{4} & \ldots & \frac{1}{n+1} \\
& & \ldots & & \\
0 & 0 & 0 & \ldots & \frac{1}{n+1}
\end{array}\right] ; \quad L_{1}^{-1}=\left[\begin{array}{cccccc}
2 & -2 & 0 & 0 & \ldots & 0 \\
0 & 3 & -3 & 0 & \ldots & 0 \\
0 & 0 & 4 & -4 & \ldots & 0 \\
\ldots & & & & & \\
0 & 0 & 0 & \ldots & n & -n \\
0 & 0 & 0 & \ldots & 0 & n+1
\end{array}\right]
$$

Also

- $L_{2}$ is obtained from $L_{1}$ by switching rows $n-1$ and $n$.

- $L_{2}^{-1}$ is obtained from $L_{1}^{-1}$ by switching columns $n-1$ and $n$.

- $L_{7}$ is obtained from $L_{1}$ by switching rows $n-4$ and $n-3$.

- $L_{7}^{-1}$ is obtained from $L_{1}^{-1}$ by switching columns $n-4$ and $n-3$.

Using these formulas one can work out, in general, that

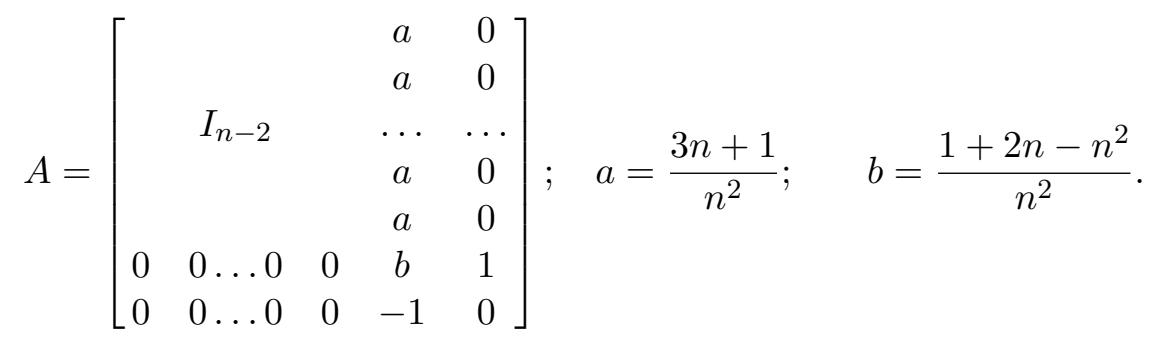

In the case $n=4$ we just compute this explicitly. For the general case, we note that both $L_{1}$ and $L_{2}$ have the same action on $\boldsymbol{R}^{n-2}$ and both preserve this subspace. Hence the first $n-2$ columns of $\left[L_{1}, L_{2}\right]^{2}$ are $e_{1}, \ldots, e_{n-2}$. Only the last two columns of $\left[L_{1}, L_{2}\right]^{2}$ need to be computed. 
For $B$ one can compute that

$$
\begin{aligned}
& B=B\left(0, \frac{n+1}{n(n-2)} ; 2-n\right)
\end{aligned}
$$

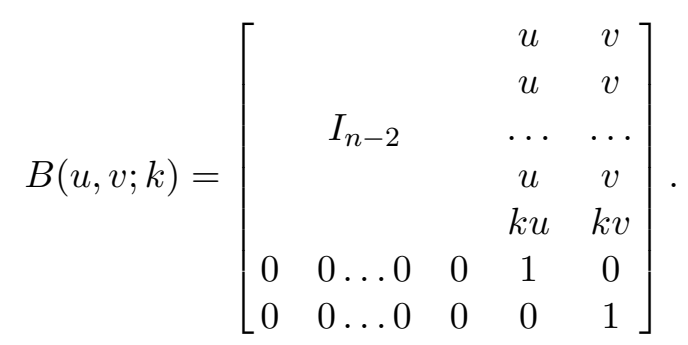

Again this can be computed explicitly in the case $n=4$.

\subsection{The Proof}

The Lie algebra to $S L_{n}(\boldsymbol{R})$ is the vector space of traceless $n \times n$ matrices. We say that $\widehat{\Omega}_{n}$ scrapes a Lie algebra element $M$ if the closure of $\widehat{\Omega}_{n}$ contains the subgroup $\exp (t M)$ generated by $M$. The set of scraped elements forms a vector space. This follows from the formula [FH, Exercise 8.38]:

$$
\exp \left(M_{1}+M_{2}\right)=\lim _{k \rightarrow \infty}\left(\exp \left(M_{1} / k\right) \cdot \exp \left(M_{2} / k\right)\right)^{k}
$$

To save words, we call the above fact the vector space property.

Let $A$ and $B$ be as above.

Lemma $5.1 A$ is an infinite order elliptic element for all $n$.

Proof: The bottom right $2 \times 2$ minor $A^{\prime}$ of $A$ has trace $n_{b} \in(-2,2)$ and hence is elliptic for all $n$. Hence $A$ is elliptic for all $n$. The only finite order elliptic elements in $S L_{2}(\boldsymbol{R})$ with rational trace have order at most 4 . The only possible traces for these elements are $-1,0,1$. None of these equals $b$. Hence $A^{\prime}$ has infinite order. Hence $A$ has infinite order.

Lemma 5.2 For any $\left(u_{1}, v_{1} ; k\right)$ there is some $\left(u_{2}, v_{2} ; k\right)$ (with the same $k$ ) such that $A B_{1} A^{-1}=B_{2}$. Here we have set $B_{j}=B\left(u_{j}, v_{j} ; k\right)$. Moreover, the pairs $\left(u_{1}, v_{1}\right)$ and $\left(u_{2}, v_{2}\right)$ are linearly independent as long as they are nonzero. 
Proof: We will give the proof in the case $n=4$. The general case is more tedious but similar. In the case $n=4$ we have:

$$
\begin{gathered}
A B_{1} A^{-1}=\left[\begin{array}{cccc}
1 & 0 & a & 0 \\
0 & 1 & a & 0 \\
0 & 0 & b & 1 \\
0 & 0 & -1 & 0
\end{array}\right]\left[\begin{array}{cccc}
1 & 0 & u_{1} & v_{1} \\
0 & 1 & k u_{1} & k v_{1} \\
0 & 0 & 1 & 0 \\
0 & 0 & 0 & 1
\end{array}\right]\left[\begin{array}{cccc}
1 & 0 & 0 & a \\
0 & 1 & 0 & a \\
0 & 0 & 0 & -1 \\
0 & 0 & 1 & b
\end{array}\right]= \\
{\left[\begin{array}{cccc}
1 & 0 & v_{1} & b v_{1}-u_{1} \\
0 & 1 & k v_{1} & k\left(b v_{1}-u_{1}\right) \\
0 & 0 & 1 & 0 \\
0 & 0 & 0 & 1
\end{array}\right]}
\end{gathered}
$$

Thus we see that

$$
\left[\begin{array}{l}
u_{2} \\
v_{2}
\end{array}\right]=\left[\begin{array}{c}
v_{1} \\
b v_{1}-u_{2}
\end{array}\right]
$$

Therefore

$$
\operatorname{det}\left[\begin{array}{ll}
u_{1} & u_{2} \\
v_{1} & v_{2}
\end{array}\right]=-\left(u_{1}^{2}+v_{2}^{2}-b u_{1} v_{2}\right)<0 .
$$

The last inequality comes from the fact that $|b|<2$.

Let $k=2-n$. Let

$$
B_{r}=A^{r} B A^{-r}=B\left(u_{r}, v_{r} ; k\right)
$$

Here $u_{r}$ and $v_{r}$ also depend on $n$, but we suppress this from our notation. A straightforward calculuation, just like the preceding lemma, shows that

$$
B_{r} B_{s}^{-1}=B\left(u_{r}-u_{s}, v_{r}-v_{s} ; k\right) .
$$

Since $A$ is an infinite order elliptic element we can choose $r$ and $s$ so that $u_{r}-u_{s}$ and $v_{r}-v_{s}$ are as close as we like to 0 . This means that $\widehat{\Omega}_{n}$, which contains all the elements we are discussing, scrapes two elements:

$$
\left[\begin{array}{ccccc} 
& & & u_{j} & v_{j} \\
& & & u_{j} & v_{j} \\
& O & & \cdots & \cdots \\
& & & u_{j} & v_{j} \\
& & & k u_{j} & k v_{j} \\
0 & 0 \ldots 0 & 0 & 0 & 0 \\
0 & 0 \ldots 0 & 0 & 0 & 0
\end{array}\right] ; \quad j=1,2 .
$$

Here $\left(u_{1}, v_{1}\right)$ and $\left(u_{2}, v_{2}\right)$ are linearly independent. The second element is obtained from the first by conjugating by $A$ and looking at the action in Equation 
38. Using these elements and the vector space property, we see that $\widehat{\Omega}_{n}$ scrapes the element

$$
\left[\begin{array}{ccccc} 
& & & 0 & 1 \\
& & & 0 & 1 \\
& O & & \ldots & \ldots \\
& & & 0 & 1 \\
& & & 0 & k \\
0 & 0 \ldots 0 & 0 & 0 & 0 \\
0 & 0 \ldots 0 & 0 & 0 & 0
\end{array}\right] .
$$

Since $\widehat{\Omega}$ contains all the permutation matrices, $\widehat{\Omega}$ scrapes the $n-2$ elements obtained by permuting the top $n-2$ rows of the preceding element. Using these elements and the vector space property, we see that $\widehat{\Omega}_{n}$ scrapes

$$
\left[\begin{array}{ccccc} 
& & & 0 & 1 \\
& & & 0 & 0 \\
& & & \ldots & \ldots \\
& & & 0 & 0 \\
0 & 0 \ldots 0 & 0 & 0 & 0 \\
0 & 0 \ldots 0 & 0 & 0 & 0
\end{array}\right] .
$$

Using the fact that $\widehat{\Omega}_{n}$ contains all the permutation matrices we see that $\widehat{\Omega}_{n}$ scrapes any element which has all zeros along the main diagonal and one nonzero element. Using the vector space property we now see that $\widehat{\Omega}_{n}$ scrapes any element with all zeros on the main diagonal. Call these elements type 1.

The density of $\widehat{\Omega}_{n}$ follows from the fact that it scrapes all type 1 elements, but we will give a more explicit proof. Looking at our element $A$ we see that $\widehat{\Omega}_{n}$ scrapes an element whose diagonal has the form $(0, \ldots, 0,1,-1)$. Since $\widehat{\Omega}_{n}$ contains all the permutation matrices, and scrapes all type 1 elements, we see that $\widehat{\Omega}_{n}$ scrapes any element which has 2 nonzero entries on the diagonal. Such elements generate the vector space of traceless matrices. Hence $\widehat{\Omega}_{n}$ scrapes every element of the Lie algebra. Hence $\widehat{\Omega}_{n}$ is dense in $S L_{n}(\boldsymbol{R})$. This completes our proof. 


\section{References}

[B] A.F. Beardon, The Geometry of Discrete Groups, GTM 91, Springer-Verlag (1983)

[BBC] I. Bárány, A.F. Beardon, T.K. Carne, Barycentric subdivision of triangles and semigroups of Mobius maps, Mathematika 43 (1996)

[FH] W. Fulton and J. Harris, Representation Theory, A First Course, GTM 129, Springer-Verlag (1991)

[S] R. Schwartz, The Density of Shapes in Three Dimensional Barycentric Subdivision, Journal of Discrete and Computational Geometry 30 (2003)

[Sp] E. Spanier, Algebraic Topology, Springer-Verlag (1966)

[W] S. Wolfram, The Mathematica Book, Fourth Edition, Cambridge University Press (1999)

Richard Evan Schwartz

Department of Mathematics

Brown University

E-mail:res@math.umd.edu 